\title{
VALIDAÇÃO EM MÉTODOS CROMATOGRÁFICOS PARA ANÁLISES DE PEQUENAS MOLÉCULAS EM MATRIZES BIOLÓGICAS
}

\author{
Neila Maria Cassiano, Juliana Cristina Barreiro, Lúcia Regina Rocha Martins, Regina Vincenzi Oliveira e \\ Quezia Bezerra Cass* \\ Departamento de Química, Universidade Federal de São Carlos, CP 676, 13560-970 São Carlos - SP, Brasil
}

Recebido em 23/4/08; aceito em 11/9/08; publicado na web em 2/2/09

\begin{abstract}
CHROMATOGRAPHIC METHODS VALIDATION FOR ANALYSIS OF SMALL MOLECULES IN BIOLOGICAL MATRICES. Chromatographic methods are commonly used for analysis of small molecules in different biological matrices. An important step to be considered upon a bioanalytical method's development is the capacity to yield reliable and reproducible results. This review discusses validation procedures adopted by different governmental agencies, such as Food and Drug Administration (USA), European Union (EU) and Agência Nacional de Vigilância Sanitária (BR) for quantification of small molecules by bioanalytical chromatographic methods. The main parameters addressed in this review are: selectivity, linearity, precision, accuracy, quantification and detection limits, recovery, dilution integrity, stability and robustness. Also, the acceptance criterions are clearly specified.
\end{abstract}

Keywords: chromatography; bioanalytical method validation; figures of merit.

\section{INTRODUÇÃO}

A validação de métodos cromatográficos para a quantificação de compostos endógenos ou exógenos em matrizes biológicas tem sido tema de discussão em conferências e artigos científicos. Representantes da comunidade científica, das indústrias e agências reguladoras do Brasil (ANVISA), EUA (US-FDA) e Europa (EU) definiram os parâmetros de validação, que posteriormente foram uniformizados pela ICH (International Conference on Harmonization).

As agências reguladoras disponibilizam guias para a validação de métodos bioanalíticos, ${ }^{1-3}$ sendo que tais documentos usualmente sugerem as diretrizes a serem seguidas e são, portanto, abertos a interpretações. Se, por um lado, para alguns analistas isto é benéfico, pois possibilita a flexibilização das condições analíticas, para outros, entretanto, tais documentos são vagos, no que se refere à forma como alguns experimentos devem ser conduzidos. Assim, alguns artigos publicados apresentam excelentes discussões sobre estratégias e modelos experimentais a serem adotados na validação de métodos bioanalíticos.

Nesse contexto, esta revisão apresenta as condutas experimentais e os critérios de aceitação para a validação de métodos bioanalíticos, para pequenas moléculas, por cromatografia líquida de alta eficiência (HPLC).

\section{PARÂMETROS ANALÍTICOS PARA VALIDAÇÃO DE MÉTODOS BIOANALÍTICOS}

Vários trabalhos na literatura descrevem os parâmetros que devem ser avaliados na validação de um método bioanalítico e, geralmente há uma concordância que estes parâmetros analíticos devem incluir: seletividade, curva de calibração (linearidade), precisão, exatidão, limite de quantificação, limite de detecção, eficiência de extração (recuperação), integridade da diluição, estabilidade e robustez. ${ }^{4-10}$

*e-mail: quezia@pq.cnpq.br

\section{Seletividade}

A seletividade e/ou especificidade de um método bioanalítico é um importante parâmetro a ser avaliado para garantir que a quantificação do analito de interesse não seja afetada pela presença de metabólitos, produtos de degradação, fármacos co-administrados ou compostos endógenos. ${ }^{4,11,12}$

A resposta que um determinado método bioanalítico apresenta para as diversas substâncias presentes em uma amostra é definida como seletividade, enquanto que a especificidade se baseia na habilidade que um determinado método apresenta em responder a uma única substância de interesse dentre outras presentes na amostra. A avaliação da seletividade é determinada não somente considerando a aplicação de um método, mas também a técnica instrumental a ser utilizada. ${ }^{8,13,14}$ Particularmente para os métodos cromatográficos, como a maioria produz respostas não só para os analitos de interesse, mas também para outros compostos presentes em uma amostra, o termo seletividade é mais apropriado que o termo especificidade. ${ }^{8}$

Os órgãos de regulamentação vigentes preconizam que um método bioanalítico só é considerado válido quando a seletividade for corretamente avaliada, juntamente com as outras figuras de mérito supracitadas. Cabe ressaltar que, caso a seletividade de um determinado método não seja assegurada, a linearidade, exatidão e precisão estarão seriamente comprometidas. ${ }^{2,4,15}$

Em geral, a seletividade de um método bioanalítico é avaliada a partir da análise da amostra branco de 6 matrizes biológicas de diferentes procedências, as quais não devem apresentar qualquer interferente, visível pelo detector em uso, na região do analito de interesse. ${ }^{2,4}$ Shah et $a l .{ }^{15}$ e Hill ${ }^{16}$ sugerem que, se uma, ou mais, amostras branco apresentarem quantidades significantes de interferentes, deve-se avaliar amostras branco adicionais e, se o interferente persistir, o método deve ser modificado.

Segundo Dadgar e Burnett, ${ }^{14}$ a presença de interferentes é considerada "inaceitável" quando afeta a exatidão das concentrações próximas aos limites de quantificação (LQ), levando a resultados errôneos. Quando os critérios de precisão e exatidão do LQ não forem atingidos, deve-se ajustar os valores de LQ para valores acima dos interferentes esperados. No entanto, este procedimento pode 
comprometer a sensibilidade e a seletividade do método, e só deve ser considerado se os valores de LQ encontrados forem maiores que as concentrações esperadas nas amostras desconhecidas.

Considerando os métodos cromatográficos, caso a resposta de uma banda cromatográfica presente na análise da amostra branco coincida com o tempo de retenção do analito de interesse, a mesma não deve se apresentar maior que $20 \%$ do limite de quantificação do analito. ${ }^{4}$ Este procedimento é, entretanto, considerado questionável, uma vez que a probabilidade de se detectar qualquer substância interferente é reduzida quando somente 6 amostras de branco são

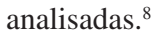

$\mathrm{O} \mathrm{OJEC}^{3}$ (Official Journal of the European Communities) recomenda que um número superior de amostras branco $(\mathrm{n} \geq 20)$ sejam avaliadas para verificar a presença de interferentes. Além disso, recomenda-se que uma concentração relevante de substâncias que normalmente interferem na identificação e/ou quantificação do analito de interesse sejam adicionadas às amostras branco. Este procedimento permite verificar após as análises, se: (i) a presença de interferentes é resultante de uma falsa identificação; (ii) a identificação do analito está sendo "mascarada" pela presença de um ou mais interferentes e, por último, se (iii) a quantificação do analito é consideravelmente influenciada pela presença de interferentes.

Segundo Bansal e Shah, ${ }^{4,5}$ dois tipos de seletividade devem ser consideradas no desenvolvimento de métodos bioanalíticos cromatográficos: (i) seletividade específica - devido à presença de substâncias interferentes que apresentam características físico-químicas similares ao analito e, (ii) seletividade não específica - também conhecida como efeito matriz, devido à presença de interferentes inerentes à matriz (por exemplo, proteínas presentes no soro, lipídeos, dentre outros).

Alguns pesquisadores ${ }^{9,14}$ sugerem que a seletividade de um método bioanalítico pode ser avaliada experimentalmente a partir das seguintes etapas: (a) análise de amostras branco de diferentes procedências, a qual é suficiente para demonstrar a presença de potenciais interferentes de substâncias comumente presentes em matrizes biológicas. No entanto, este procedimento não fornece informações sobre os interferentes provenientes do metabolismo da substância de interesse; (b) adição dos metabólitos conhecidos e produtos de degradação, ou qualquer outro interferente, obtidos comercialmente ou sintetizados, na amostra branco; (c) produção dos principais metabólitos in vitro, e posterior análise com o intuito de verificar potenciais interferentes com os analitos de interesse; (d) avaliar o efeito dos principais produtos gerados por hidrólise ácida no estômago, a partir da incubação in vitro do analito em meio ácido seguida por neutralização, e posterior análise com o intuito de verificar potencias interferentes com os analitos de interesse; (e) análise de amostra branco preparada em solvente, com verificação da seletividade em relação aos interferentes exógenos, utilizando o mesmo volume, reagentes e equipamentos. Caso seja verificada a presença de interferentes, deve-se identificar a fonte de contaminação.

A ANVISA $^{2}$ recomenda que sejam realizados testes em amostras da matriz biológica (sangue, plasma, soro, urina, etc) obtidas de 6 indivíduos. Considerando a matriz plasma e soro, devem-se utilizar quatro amostras normais, uma lipêmica e uma hemolisada, sob condições controladas referentes ao tempo, alimentação e outros fatores importantes para o estudo. Cada amostra branco deve ser testada utilizando o procedimento e as condições cromatográficas propostas, sendo os resultados comparados com aqueles obtidos em solução aquosa do analito, na concentração próxima ao LQ. Qualquer amostra branco que apresentar interferência significativa no tempo de retenção do fármaco, metabólito ou padrão interno deve ser rejeitada. Caso uma ou mais amostras apresentem interferentes, novas amostras de outros 6 indivíduos devem ser testadas. Se os interferentes apresentarem-se significativos no tempo de retenção do fármaco para este novo grupo, o método deve ser alterado visando eliminá-lo. Segundo a ANVISA, as respostas dos picos interferentes no tempo de retenção dos fármacos e do padrão interno devem ser inferiores, respectivamente, a 20 e 5\% da resposta na concentração utilizada.

Para os métodos cromatográficos, deve-se tomar as precauções necessárias para garantir a pureza das bandas cromatográficas. A utilização de testes de pureza da banda (por exemplo, com auxílio de detector de arranjo de fotodiodos ou espectrometria de massas) é fundamental para demonstrar que a banda cromatográfica é atribuída a um só componente.

Uma vez definidos e minimizados os interferentes provenientes de metabólitos e produtos de degradação durante o desenvolvimento do método bioanalítico, a etapa seguinte consiste em avaliar a presença de substâncias não monitoradas e que são provenientes da matriz biológica, afetando a detecção do analito de interesse, o qual é conhecido como efeito de matriz. ${ }^{4,14}$

\section{Efeito de matriz}

A investigação do efeito de matriz durante a quantificação de compostos endógenos ou exógenos e/ou seus metabólitos é um importante parâmetro a ser avaliado durante o desenvolvimento e validação de um método bioanalítico. Este efeito ocorre quando substâncias inerentes à matriz biológica coeluem com os compostos de interesse, sendo alvo de especulação em métodos bioanalíticos convencionais, tais como: HPLC/FLU, HPLC/UV, HPLC/EC e mais recentemente em HPLC-MS/MS. ${ }^{17}$ Este último, em virtude da elevada seletividade e especificidade quando na presença de matrizes biológicas, apresenta-se propenso a erros, uma vez que a presença de substâncias não monitoradas e que coeluem da matriz podem afetar a detecção dos analitos comprometendo a eficiência de ionização dos mesmos, caracterizando assim o efeito de matriz. Este fenômeno foi primeiramente descrito por Tang e Kebarle, ${ }^{18}$ que mostraram que a resposta para bases orgânicas em uma determinada matriz, utilizando a fonte de ionização electrospray, diminuiu à medida que a concentração de outras bases orgânicas foi aumentada. O mecanismo exato do efeito de matriz é desconhecido, mas, provavelmente, é originado da competição entre um analito e a coeluição de um componente da matriz não monitorado. ${ }^{19}$

King et al..$^{20}$ mostraram, a partir de uma série de experimentos, que os efeitos de matriz são resultantes da competição entre o analito e componentes não voláteis presentes na matriz, pela transferência de elétrons do capilar para a solução, e a separação de cargas na superfície das gotas que irão dar origem aos íons em fase gasosa. ${ }^{21}$ Dependendo do ambiente no qual a ionização e o processo de evaporação estejam ocorrendo, esta competição deve efetivamente diminuir (supressão) ou aumentar a eficiência de formação dos íons do analito de interesse, que se apresentam, inicialmente, nas mesmas concentrações na interface. Sendo assim, a eficiência de formação dos íons do analito é fortemente dependente da natureza da matriz na fonte de ionização. Bonfiglio et $a l .{ }^{22}$ reportaram que a natureza química da substância a ser analisada tem um efeito significativo no grau do efeito de matriz. Em um estudo com quatro compostos de diferentes polaridades, sob as mesmas condições espectrométricas, o mais polar apresentou uma elevada supressão de íons em relação ao menos polar. Neste caso, a diferença de ionização entre os compostos pode comprometer a quantificação dos mesmos, sendo necessário o uso de padrão interno. ${ }^{19}$

A medida de quantificação do efeito de matriz fornece informações adicionais na validação de métodos bioanalíticos baseados na espectrometria de massas. Sendo assim, Viswanathan et al. ${ }^{23}$ propuseram que o efeito de matriz pode ser expresso a partir de um fator denominado fator matriz (FM) calculado a partir de 6 amostras de uma mesma matriz biológica de diferentes procedências, sendo definido como: 


$$
\mathrm{FM}=\frac{\text { resposta do analito na presença da matriz }}{\text { resposta do analito na ausência da matriz }}
$$

onde a resposta do analito é definida como a área, altura, razão de área ou razão da altura da banda cromatográfica. Quando se utiliza o método de quantificação por padronização interna, a razão de área (ou altura) da banda é expressa como a razão de área (ou altura) da banda para o analito versus a do padrão interno.

Quando o valor de FM é igual a 1, significa que nenhum efeito de matriz é observado, FM menor que 1 sugere supressão de ionização, enquanto que FM maior que 1 pode ser causado por um aumento na ionização. Um FM absoluto de cerca de 1 não significa que o método bioanalítico seja confiável. No entanto, valores de FM variáveis podem ser causados pela falta de precisão da análise. A variabilidade de FM deve apresentar coeficiente de variação menor que $15 \%$.

A intensidade do efeito de matriz, utilizando o método HPLCMS/MS, pode ser avaliado de duas maneiras: adição pós-extração e infusão pós-coluna. No modo de adição pós-extração, as respostas obtidas para as amostras cujos analitos de interesse foram adicionados pós-extração são comparadas com as soluções preparadas na fase móvel, sob as mesmas condições. A diferença de resposta entre a amostra pós-extração e a solução preparada em solvente, dividida pela resposta da solução em solvente determina o grau do efeito de matriz. Esta análise é considerada estática, pois fornece somente a informação do efeito de matriz no ponto de eluição do analito, enquanto que a infusão pós-coluna (Figura 1) é considerada dinâmica na determinação do efeito de matriz. Neste modo, uma bomba de infusão é usada para produzir uma vazão constante do analito. A infusão é feita no eluente após a coluna cromatográfica e antes da fonte de ionização do espectrômetro de massas. A matriz extraída (sem adição do analito) é cromatografada, na condição cromatográfica desenvolvida, e a resposta do analito infuso é registrada. ${ }^{19,24} \mathrm{~A}$ infusão pós-coluna permite verificar a influência da matriz na resposta do analito. No entanto, a concentração do analito infuso deve ser na mesma faixa de concentração a ser cromatografado. Caso contrário, a habilidade da fonte de ionização em gerar íons é dificultada e pode levar a resultados errôneos.

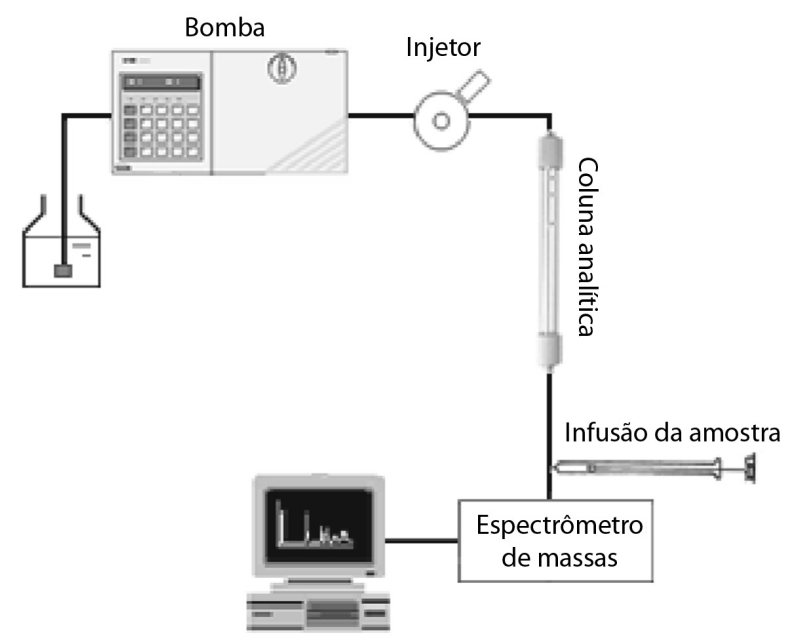

Figura 1. Sistema de infusão pós-coluna

Os experimentos de infusão pós-coluna permitem avaliar: i) a influência de diferentes modos de preparo de amostras no efeito de matriz; ii) a adequação da coluna analítica, uma vez que o efeito é avaliado após passar por toda a coluna cromatográfica; iii) aspectos mecanísticos dos efeitos de matriz e, iv) a influência de aditivos móveis na resposta. ${ }^{19}$
Vários métodos de preparo de amostras biológicas têm sido descritos na literatura para análises por HPLC. ${ }^{25-26}$ A precipitação de proteínas usando solvente orgânico apresenta um efeito de matriz maior, quando comparada à extração em fase sólida e à extração líquido-líquido. Mais recentemente, a cromatografia bi-dimensional tem demonstrado um efeito mínimo de matriz. ${ }^{27,28}$ No entanto, a escolha do modo de preparo das amostras é feita levando-se em consideração suas características físico-químicas e sua estabilidade, a separação cromatográfica obtida durante a otimização do método e os níveis de recuperação. . $^{29,30}$

\section{Curva de calibração (linearidade)}

A curva de calibração corresponde ao modelo matemático que estabelece uma relação entre a resposta instrumental (área/altura da banda cromatográfica) e a concentração do analito. É necessário elaborar uma curva de calibração para cada analito que se deseja determinar e para cada corrida analítica. ${ }^{1,2}$

O modelo de calibração deve ser construído a partir da análise de, no mínimo, 6 a 8 concentrações conhecidas do analito (padrões de calibração) adicionadas na mesma matriz biológica para a qual o método foi desenvolvido e será aplicado (superposição de matriz). ${ }^{31}$ As concentrações estabelecidas devem contemplar o intervalo de variação esperado, desde o LQ até $120 \%$ da máxima concentração que se pretende analisar. ${ }^{1,2}$ Além disso, deve-se realizar análise da amostra branco (matriz isenta da adição do analito e do padrão interno, quando for o caso) e da amostra zero (matriz adicionada do padrão interno, quando for o caso).

Os critérios para aceitação da curva de calibração seguem normas estabelecidas por guias de validação publicados por agências reguladoras oficiais. De acordo com as normas atualmente em vigor, ${ }^{1,2}$ aceita-se um coeficiente de variação menor ou igual a $20 \%$ em relação à concentração nominal para o LQ e menor ou igual a 15\% para as demais concentrações. Além disso, o coeficiente de correlação linear ( $r$ ) deve ser igual ou superior a 0,98 .

\section{Métodos de padronização}

Pode-se optar por três diferentes tipos de padronização para a construção da curva de calibração. Isto é feito de acordo com o tipo de análise e do tratamento utilizado para a amostra. O método de padronização escolhido deve fornecer a melhor exatidão possível, além de um alto nível de precisão. ${ }^{31}$

A padronização externa é utilizada para amostras que não precisam de extenso pré-tratamento; os padrões de calibração são obtidos pela adição de concentrações conhecidas do analito na matriz. Ao ser aplicado para análises de amostras desconhecidas, esse método compara a área do pico do analito a ser quantificado com as áreas obtidas a partir dos padrões de calibração (através do gráfico de concentração versus resposta ou pela equação da curva resultante) (Figura 2a). ${ }^{32-34}$

A padronização interna consiste na preparação dos padrões de calibração contendo diferentes concentrações do analito, nos quais se adiciona uma concentração fixa do padrão interno (PI). Esse método permite avaliar a variação da resposta em função de manipulações da amostra (por exemplo, concentração, extração, preparo da amostra), sendo o método de escolha para análises em matrizes biológicas, quando não se trabalha com injeção direta de amostras. O modelo de regressão e o gráfico são elaborados pela relação entre a razão das áreas (área do analito/área do padrão interno) com as concentrações do analito. Na aplicação do método, as amostras desconhecidas são analisadas após a adição da mesma quantidade do padrão interno (Figura $2 b$ ). ${ }^{32}$

A padronização por adição de padrão é o método utilizado nas seguintes situações: quando não é possível obter a matriz isenta do analito; em matrizes muito complexas; quando há fortes interações 
entre o analito e a matriz e quando é difícil encontrar um padrão interno adequado. ${ }^{31}$ Este método consiste na adição de diferentes concentrações do analito à matriz, que já contém uma quantidade desconhecida do mesmo. A adição deve ser feita antes do processo de tratamento da amostra. A concentração do analito na matriz biológica é determinada pela extrapolação da reta, definida pelas demais concentrações analisadas (Figura 2c). ${ }^{31,35}$

Considerando que, em geral, os métodos bioanalíticos utilizam etapas de tratamento com significativa manipulação da amostra (por exemplo, extração, diluição, centrifugação, concentração, etc), na maioria das vezes utiliza-se a padronização interna para a elaboração da curva de calibração.

\section{a}

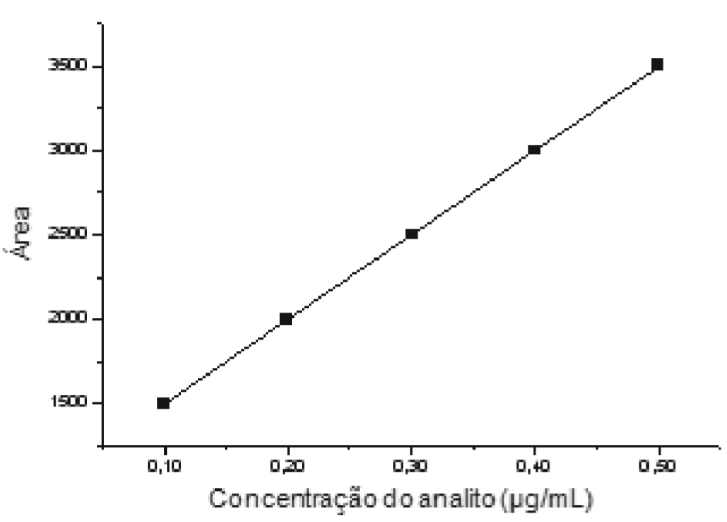

b

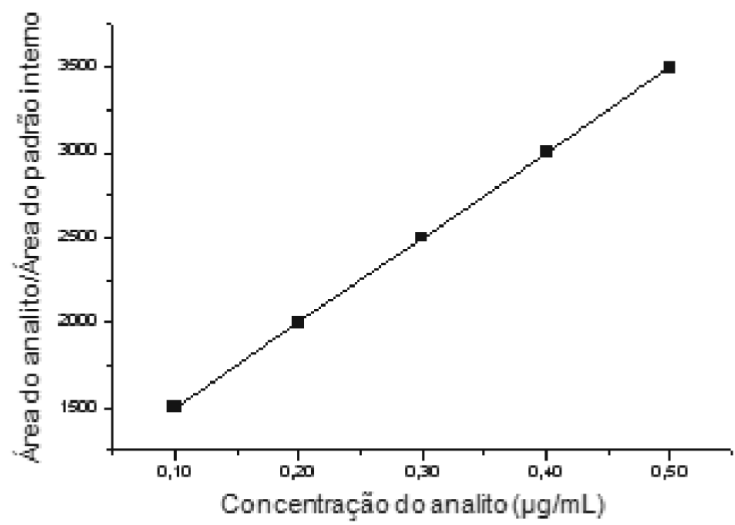

C

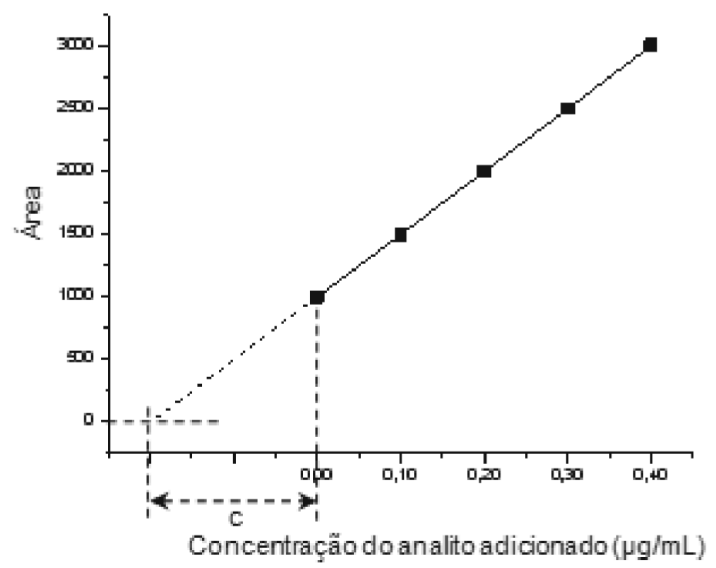

Figura 2. Métodos de padronização para curva de calibração. a) padrão externo; b) padrão interno e c) adição de padrão
Construção e avaliação de modelos de regressão

Para que um método bioanalítico por HPLC seja adequadamente validado e os dados gerados sejam confiáveis, é essencial obter uma curva de calibração bem elaborada e interpretada. ${ }^{8,36}$ As concentrações dos analitos são determinadas por meio de regressões obtidas a partir das curvas de calibração e, embora em alguns casos seja necessária a utilização de calibração não-linear, normalmente se opta pela regressão linear, por sua maior simplicidade e aplicabilidade. .,37 $^{\text {a }}$

A elaboração de um modelo de regressão pressupõe a existência de um conjunto de pontos que apresentam uma tendência regular e que podem ser representados por uma função matemática, que normalmente é escolhida através de um processo de ajuste conhecido como método dos mínimos quadrados. Portanto, estabelece-se a tendência da variável dependente $(y)$ em função da variável independente $(x)$ que, para uma regressão linear, pode ser expressa como $y=a x+b$, onde $a$ é o coeficiente angular da reta e $b$ seu intercepto com o eixo $y$ (ou coeficiente linear).

Na construção da curva de calibração, para cada concentração avaliada $\left(x_{i}\right)$ é obtida uma determinada área/altura de banda cromatográfica $\left(y_{i}\right)$, resultando então em um conjunto de $\mathrm{N}$ pontos caracterizados pelas coordenadas $\left(x, y_{i}\right)$, a partir dos quais se estabeleceu $y=f(x)$. Para cada valor de $x$, pode-se estimar um valor para $y$, denominado $y_{\text {est }}$ O método de mínimos quadrados sugere que $y_{\text {est }}$ seja obtido de tal forma que a diferença em relação ao conjunto de dados experimentais $\left(y_{i}\right)$ seja mínima, ou seja:

$$
\mathrm{SQr}=\sum\left(y_{i}-y_{e s t}\right)^{2}
$$

sendo SQr a soma dos quadrados dos resíduos obtidos entre os valores experimentais e estimados. ${ }^{38}$ Para um entendimento mais pormenorizado dos cálculos matemáticos que envolvem a construção da regressão por mínimos quadrados, podem ser consultadas as refs. 39 a 42 .

O coeficiente de correlação $r$, variável entre -1 e +1 , representa o nível de erro em relação ao sinal analítico e a qualidade de ajuste da equação à curva de calibração. Em muitos casos, o conceito estatístico de $r$ é mal interpretado, sendo utilizado como critério de aceitação da linearidade da regressão para muitos analistas. Porém, mesmo quando se obtém valores de $r$ próximos a 1 ou -1, é possível que a regressão não seja linear ou que o modelo de regressão linear proposto não atenda a faixa de concentração estabelecida. ${ }^{37,39}$

Pode-se verificar se a equação de regressão linear é estatisticamente significativa através dos seguintes testes: ajuste do modelo linear, validade da regressão, eficiência da regressão e eficiência máxima.

\section{Teste de ajuste do modelo linear}

A curva de calibração é construída pela análise de pelo menos 6 concentrações ao longo da faixa de trabalho, com replicatas para cada concentração. É importante que as repetições de análises sejam realizadas a partir de replicatas autênticas, ou seja, cada resposta instrumental (área/altura de banda) deve ser o resultado da repetição de todo o processo de análise da amostra, desde sua preparação inicial, não sendo suficiente apenas reintroduzir a mesma amostra no sistema cromatográfico. ${ }^{1,2}$

A média dos valores encontrados para cada concentração é o valor aceito e o desvio padrão da média é calculado para cada concentração, portanto, as incertezas (erros aleatórios) não são representativas para a curva de calibração como um todo, pois se tratam de incertezas pontuais. A dispersão das medidas (valores de y) deve ser independente da concentração do padrão de calibração, seguindo uma distribuição normal, propriedade conhecida como homoscedasticidade. ${ }^{36-40}$ Porém, quando o intervalo de concentração 
é muito amplo (como ocorre em vários métodos bioanalíticos), maiores desvios serão encontrados nas concentrações mais altas, o que poderá influenciar a regressão linear mais intensamente que os pequenos desvios ocasionados nas concentrações mais baixas (heteroscedasticidade). ${ }^{37}$

A homoscedasticidade da regressão linear pode ser avaliada pelo teste de ajuste do modelo, pela observação do gráfico de resíduos versus concentração ${ }^{1}$ ou pela aplicação do seguinte teste $F$ de significância: ${ }^{36}$

$$
F_{\exp }=\frac{s_{2}^{2}}{s_{1}{ }^{2}}
$$

onde $F_{\exp }$ expressa a razão entre as variâncias obtidas na menor concentração $\left(s_{1}^{2}\right)$ e na maior concentração $\left(s_{2}^{2}\right)$. Compara-se esse valor ao $F_{\text {tab }}=F_{(f 1, f 2)}$ com $99 \%$ de confiança, sendo $f 1=f 2=(n-1)$ graus de liberdade ( $\mathrm{n}=$ número total de observações). Se a variância for constante em toda a faixa da curva de calibração, os resíduos estarão aleatoriamente distribuídos no eixo $x$ e $F_{\text {exp }}$ será menor que $F_{\text {tab }}{ }^{36}$

A avaliação de ajuste do modelo linear pela análise das variâncias (ou teste-F de significância) é um método bastante conhecido e bem estabelecido, cuja discussão está fora do contexto deste trabalho. ${ }^{41,42}$ Para esse teste, utilizam-se os termos detalhados na Tabela 1, que pode ser aplicada a modelos com $p$ parâmetros (neste caso, $p=2$ ). Faz-se o cálculo da razão das médias quadráticas, ou seja, $F=$ MQfa/MQep e compara-se o valor obtido com $F_{\text {crítico }}=F_{(\mathrm{m}-2):(\mathrm{n}-\mathrm{m})}$ (tabelado), para o grau de confiança desejado. Se $F \leq F_{\text {crítico }}$, a linearidade é aceita e o ajuste do modelo é considerado satisfatório, ou seja, as variâncias em $y$ são independentes das concentrações em $x$.

Tabela 1. Termos calculados para análise pela variância do ajuste do modelo e da validade da regressão linear pelo método dos mínimos quadrados. Adaptada da ref. 38

\begin{tabular}{lcccc}
\hline Fonte & $\begin{array}{c}\text { Soma } \\
\text { Quadrática }\end{array}$ & Equação & $v$ & $\begin{array}{c}\text { Média } \\
\text { Quadrática }\end{array}$ \\
\hline $\begin{array}{l}\text { Regressão } \\
\text { (R) }\end{array}$ & SQR & $\sum \sum\left(y_{\text {est }}-\bar{y}\right)^{2}$ & $p-1$ & $\begin{array}{c}\mathrm{MQR}=\mathrm{SQR} / \\
(p-1)\end{array}$ \\
$\begin{array}{l}\text { Resíduo } \\
\text { r) }\end{array}$ & SQr & $\sum \sum\left(y_{i}-y_{\text {est }}\right)^{2}$ & $n-p$ & $\begin{array}{c}\mathrm{MQr}=\mathrm{SQr} / \\
(n-p)\end{array}$ \\
$\begin{array}{l}\text { erro } \\
\text { puro (ep) } \\
\text { falta de } \\
\text { ajuste (fa) }\end{array}$ & SQep & $\sum \sum\left(y_{i}-\bar{y}_{i}\right)^{2}$ & $n-m$ & $\begin{array}{c}\text { MQep }=\mathrm{SQep} / \\
(n-m)\end{array}$ \\
\begin{tabular}{l} 
Total (T) \\
\hline
\end{tabular} & SQfa & $\sum \sum\left(y_{\text {est }}-\bar{y}_{i}\right)^{2}$ & $m-p$ & $\begin{array}{c}\mathrm{MQfa}=\mathrm{SQfa} / \\
(m-p)\end{array}$ \\
& SQT & $\sum \sum\left(y_{i}-\bar{y}\right)^{2}$ & $n-1$ & $\begin{array}{c}\mathrm{MQT}=\mathrm{SQT} / \\
(n-1)\end{array}$ \\
\hline
\end{tabular}

$n=$ número total de observações; $m=$ número de níveis distintos; $p$ = número de parâmetros do modelo $(=2) ; y_{i}=$ valores experimentais de $y ; y_{\text {est }}=$ valores calculados de $y ; \bar{y}=$ média global dos valores experimentais de $y_{i} ; \bar{y}_{i}=$ média dos valores obtidos no nível $i ; \mathrm{v}=$ graus de liberdade.

Quando o modelo linear apresenta falta de ajuste, algumas opções podem ser consideradas: a) um novo intervalo de trabalho pode ser estabelecido, normalmente excluindo-se a concentração mais alta. Obtém-se um novo modelo linear de regressão, que novamente deverá ser testado quanto às variâncias $;{ }^{39}$ b) ajusta-se um modelo quadrático para o mesmo conjunto de valores e avalia-se novamente seu ajuste. Esse ajuste pode ser necessário quando do uso de alguns detectores ${ }^{43}$ ou ser uma indicação de problemas com o método bioanalítico, tais como solubilidade do analito ou supressão de íons $;{ }^{37}$ c) transformam-se os dados de $x$ e/ou $y$ antes de construir a regressão linear. Duas formas comuns são as transformações por cálculo do logaritmo ou da raiz quadrada de ambos os valores $(x$ e y) antes da regressão linear. A transformação dos dados é uma boa opção de melhorar o ajuste do modelo linear e assegurar sua robustez, uma vez que reduz significativamente o intervalo da curva de calibração, causando uma compressão dos valores e estabilizando a variância em toda a extensão das concentrações, ${ }^{37}$ d) uso de fator peso - um método pra contornar a heteroscedasticidade é aplicar um modelo de regressão ponderado, cujo princípio é dar maior importância para dados com baixa variância e menor importância para dados com alta variância. Os pesos $\left(w_{i}\right)$ comumente usados são os valores empíricos $1 / \mathrm{x}, 1 / \mathrm{x}^{2}, 1 / \mathrm{x}^{1 / 2} \mathrm{e} 1 / \mathrm{y}^{1 / 2}$, de tal forma que se calcula, para cada valor de $w_{i}$, novos valores para os coeficientes da regressão $(a$ e $b$ ) e, posteriormente, avalia-se qual dos pesos forneceu um modelo linear com melhor ajuste, calculando-se a percentagem de erro relativo individual e escolhendo o peso correspondente ao menor valor. ${ }^{36,41}$

\section{Avaliação da validade da regressão}

Para a avaliação da validade da regressão, faz-se novamente um teste $\mathrm{F}$ de significância, através dos termos descritos na Tabela 1. Calcula-se $F=\mathrm{MQR} / \mathrm{MQr}$ e compara-se com o valor de $F_{\text {crítico }}$ (tabelado), sendo $F_{\text {crítico }}=F_{(p-1),(\mathrm{n}-2)}$, no nível de confiança selecionado. Se F $\geq \mathrm{F}_{\text {crítico }}$, há indicação de que a regressão é significativa, pois aceitase que $a \neq 0$, ou seja, a inclinação da reta não é nula. Se $\mathrm{F} \leq \mathrm{F}_{\text {crítico, }}$, não há indicação da relação de linearidade entre $x$ e $y$ e, portanto, a regressão linear não tem sentido. Quanto maior for o valor de $F$, mais significativa será a relação entre $x$ e $y .{ }^{39}$

Aceitando-se a validade da regressão, pode-se calcular sua eficiência através do coeficiente de determinação, ${ }^{42}$ dado por:

$$
\mathrm{R}^{2}=\frac{\mathrm{SQR}}{\mathrm{SQT}}
$$

Valores abaixo de $0,95\left(\mathrm{R}^{2}<0,95\right)$ indicam que a regressão não é eficiente; o valor desejado para $\mathrm{R}^{2}$ é teoricamente 1 , o que ocorrerá se toda variação de $y$ em torno da média for explicada pela regressão, ou seja, não houver resíduo algum. ${ }^{42} \mathrm{~A}$ eficiência máxima da regressão (máxima variância que pode ser explicada pela regressão) é calculada pela seguinte equação: ${ }^{39}$

$$
\mathrm{R}^{2}=\frac{\mathrm{SQT}-\mathrm{SQep}}{\mathrm{SQT}}
$$

Para a elaboração de uma curva de calibração que realmente atenda às necessidades que o método proposto pretende, recomendase que sejam tomados todos os cuidados experimentais necessários para que a coleta de dados seja precisa e eficiente. Ao construir o modelo de regressão, deve-se avaliar a homoscedasticidade dos dados, ajustando-se um modelo linear (inicialmente) e procedendo-se os testes para avaliar a qualidade do modelo, através da análise pela variância. Se não houver falta de ajuste e se a regressão for válida, pode-se determinar o intervalo de confiança do modelo e fazer a regressão inversa, através da qual se calcula a concentração a partir dos valores instrumentais obtidos: ${ }^{42}$

$x=\frac{y-b}{a}$

Caso contrário, podem-se fazer novos ajustes no modelo (restrição da faixa de concentração, ${ }^{39}$ aplicação de transformações ou modificações do modelo proposto) e assim obter melhores resultados para aplicação prática do método analítico. ${ }^{42}$ 


\section{Precisão e exatidão (bias)}

A precisão e a exatidão determinam os erros de uma medida analítica e são os principais critérios usados para julgar a qualidade de um método bioanalítico.

\section{Precisão}

A precisão de um método bioanalítico é a medida dos erros aleatórios e representa a proximidade dos resultados obtidos a partir de medidas independentes de amostragens múltiplas de uma amostra homogênea. ${ }^{1,2}$ Este é um importante parâmetro que possibilita decidir se o método bioanalítico é confiável ou não para o objetivo da análise.

A precisão pode ser expressa como uma estimativa do desvio padrão (s) ou desvio padrão relativo (DPR), também conhecido como coeficiente de variação (CV\%), de uma série de repetições da mesma amostra, em diferentes preparações: ${ }^{2,6,7}$

$s=\sqrt{\frac{\left(x_{i}-\bar{x}\right)^{2}}{N-1}}$

DPR $(\%)$ ou CV $(\%)=\frac{s}{\bar{x}} \times 100$

onde $x_{\mathrm{i}}$ é o valor individual de uma determinação, $\bar{x}$ é a média aritmética das determinações e $\mathrm{N}$ é o número de determinações.

A precisão deve ser avaliada de três maneiras: ${ }^{2}$ através da repetibilidade (precisão intra-dia); precisão intermediária (precisão inter-dias) e reprodutibilidade (precisão inter-laboratorial).

\section{Repetibilidade (precisão intra-dia)}

Define a precisão do método em repetir, em um curto intervalo de tempo, os resultados obtidos nas mesmas condições de análise, ou seja, com o mesmo analista, com o mesmo equipamento, no mesmo laboratório e fazendo uso dos mesmos reagentes.

A repetibilidade não deve ser confundida com a análise da precisão instrumental ou repetibilidade de injeções de uma mesma amostra. ${ }^{44}$ É importante ressaltar que a precisão do método avaliada através da repetibilidade é investigada pela análise das amostras controle de qualidade preparadas em replicatas para cada concentração, enquanto que a precisão instrumental avalia uma sequiência de, no mínimo, 10 injeções de uma única amostra. ${ }^{31}$

\section{Precisão intermediária (precisão inter-dias)}

Define a habilidade do método em fornecer os mesmos resultados quando as análises são conduzidas no mesmo laboratório, mas em diferentes dias, por diferentes analistas e diferentes equipamentos. Para a determinação da precisão intermediária, recomenda-se um mínimo de 2 dias diferentes com analistas diferentes ${ }^{2}$ e, na maioria das validações bioanalíticas, adotam-se três dias não consecutivos.

\section{Reprodutibilidade (precisão inter-laboratorial)}

O termo é utilizado para demonstrar a precisão entre laboratórios. Os resultados são obtidos usando o mesmo método e mesmas amostras em diferentes laboratórios, diferentes analistas e equipamentos. No entanto, é possível avaliar a reprodutibilidade parcialmente, onde somente alguns dos fatores citados são modificados. Neste caso, as variáveis avaliadas devem ser registradas.

O termo reprodutibilidade é freqüentemente utilizado erroneamente para expressar as análises conduzidas em um mesmo laboratório, ou seja, para expressar a precisão intermediária, o que deve ser evitado.

De acordo com Hartmann et al., ${ }^{8}$ a investigação interlaboratorial não é necessária em estudos bioanalíticos quando a precisão interme- diária foi previamente avaliada ou, ainda, quando o método não será utilizado por diferentes laboratórios. A ANVISA² também não exige que a reprodutibilidade seja avaliada para a concessão de registros.

De acordo com as diretrizes do $\mathrm{ICH},{ }^{45}$ a precisão deve ser investigada com o uso de amostras certificadas ou, quando isto não é possível, podem ser empregadas amostras preparadas artificialmente, ou seja, amostras biológicas adicionadas com o padrão. Similarmente, o FDA ${ }^{1}$ recomenda que as amostras para a validação do método (padrões de calibração e amostras controle de qualidade) sejam preparadas na própria matriz e que os padrões sejam de referência analítica, com conhecida identificação e pureza. Três tipos de padrões de referência podem ser adotados: ${ }^{1}$ (1) amostras padrões certificadas (por exemplo, USP); (2) padrões de referência obtidos comercialmente e, (3) outros materiais de pureza conhecida, como os obtidos sinteticamente por laboratórios analíticos, desde que os materiais tenham sido previamente caracterizados e identificados.

Dentro desta última classificação descrita pelo FDA, ${ }^{1}$ entende-se que produtos naturais obtidos a partir de extratos de plantas também podem ser utilizados como padrões de referência e, da mesma forma que os padrões obtidos sinteticamente, estes compostos devem ser purificados, caracterizados e identificados.

No trabalho realizado por Beltrame et $a l .{ }^{46}$ o extrato hidroalcoólico preparado de Trichilia catigua foi inicialmente purificado por cromatografia contra-corrente. Posteriormente, a fração clorofórmica foi purificada por extração em fase sólida e cromatografia semipreparativa. Este procedimento permitiu a obtenção do composto Cinchonain Ib puro, que foi caracterizado e posteriormente utilizado para a quantificação deste mesmo composto em formulações herbais vendidas como Catuaba, no Brasil.

\section{Exatidão (bias)}

A exatidão representa a concordância entre os resultados obtidos pelo método e os valores nominais, aceitos como referência. ${ }^{1,2}$

A exatidão de um método bioanalítico é a medida dos erros aleatórios e sistemáticos (bias). ${ }^{8}$ No entanto, o termo exatidão é freqüentemente empregado para descrever somente os erros sistemáticos do método em questão e é melhor expresso com o desvio em porcentagem bias do valor de referência: ${ }^{13}$

Exatidão $(\%$ bias $)=\left(\frac{x_{i}-x}{x}\right) \times 100$

onde $x_{\mathrm{i}}$ é o valor de concentração experimental e $x$ é o valor de concentração nominal da determinação.

É importante salientar que, na validação de métodos bioanalíticos, a exatidão é obtida através da aproximação de resultados, ou seja, devido à impossibilidade de se trabalhar com amostras certificadas do analito na matriz de interesse, as amostras padrão são obtidas pela adição do analito na amostra branco. Desta forma, a exatidão é determinada para cada concentração dos três controles de qualidade através da concordância das concentrações medidas e nominais do analito na amostra adicionada. ${ }^{13}$

Assim como a precisão, a exatidão também é um parâmetro que permite julgar a confiabilidade do método bioanalítico e deve ser investigada após a determinação da seletividade, linearidade e precisão do método analítico. ${ }^{1}$

Os guias do $\mathrm{FDA}^{1}$ e da $\mathrm{ANVISA}^{2}$ preconizam que a precisão e a exatidão devem ser investigadas pela análise de, no mínimo, 3 controles de qualidades (CQ) analisados em 5 replicatas de preparo de amostras, com níveis de concentrações baixa (CQB), média (CQM) e alta (CQA) em relação ao intervalo de concentração da curva de calibração.

As amostras controle de qualidade devem ser preparadas na mesma matriz biológica para a qual o método foi desenvolvido. ${ }^{15}$ Desta 
forma, as amostras controle de qualidade devem ser submetidas às mesmas etapas de pré-tratamento utilizadas no preparo das amostras da curva de calibração e das amostras desconhecidas.

É recomendado, para o CQB, um valor de concentração igual a três vezes à do limite inferior de quantificação (LIQ); para o CQM, um valor de concentração próximo à central da curva de calibração e, para o CQA, uma concentração próxima ao valor mais alto da curva de calibração. ${ }^{1,15}$ A ANVISA, ${ }^{2}$ de modo mais específico, relata a mesma consideração do $\mathrm{FDA}^{1}$ para o valor de $\mathrm{CQB}$, enquanto propõe para o CQM uma concentração igual a aproximadamente a média entre o valor de CQB e CQA e, para o CQA, um valor de concentração que represente 75 a $90 \%$ do maior valor da curva de calibração.

O protocolo de validação utilizado por pesquisadores do nosso grupo descreve valores para o CQB de 110-120\% em relação ao ponto de menor concentração da curva de calibração; para o CQM de 40-60\% em relação ao ponto de maior concentração da curva de calibração e para o CQA valores de 75-95\% em relação ao ponto de mais alta concentração da curva de calibração. $27,28,33,34,47,48$

A precisão e a exatidão, determinadas para cada uma das concentrações da curva de calibração e dos controles de qualidade, devem estar no intervalo de $\pm 15 \%$ da concentração nominal de todas as concentrações, exceto para o valor de LIQ, que não deve ultrapassar $20 \%$. No trabalho de Hartmann et al. ${ }^{8}$ o significado dos limites de aceitação de 15 e $20 \%$ é discutido em detalhes.

De acordo com Bansal e DeStefano, ${ }^{4}$ amostras controle de qualidade que, após as análises, apresentaram desvios superiores aos limites aceitáveis, somente devem ser excluídas dos cálculos de precisão e exatidão quando os erros encontrados forem provenientes de problemas conhecidos, como cromatográficos ou de extração.

Estes critérios de aceitação para a precisão e exatidão de métodos bioanalíticos têm sido amplamente aceitos e utilizados..$^{5,15,23,49}$ No entanto, variam consideravelmente as propostas sobre o número de controles de qualidade e de replicatas de cada concentração que devem ser utilizadas nas validações dos métodos bioanalíticos. ${ }^{8,13,50}$

\section{Limites de quantificação (LQ) e de detecção (LD)}

Os termos limite de quantificação (LQ) e limite de detecção (LD) são utilizados para demonstrar a habilidade do método em quantificar/ detectar baixas concentrações de um analito.

O limite de detecção é definido como a menor concentração de um analito que o método é capaz de diferenciar confiavelmente do ruído de fundo. ${ }^{2,15}$ É importante ressaltar que esta concentração é detectada com segurança porém, em contrapartida, não pode ser quantificada.

A ANVISA $^{2}$ recomenda que o LD seja de 2 a 3 vezes superior ao ruído da linha de base. No entanto, é muito comum o registro de vários picos cromatográficos nas amostras branco, o que pode ocasionar interferência no sinal de fundo ou, até mesmo, uma estimativa errada do ruído da linha de base e, conseqüentemente, do valor de $\mathrm{LD}$, o que dificulta a comparação de valores obtidos para limites de detecção entre diferentes laboratórios. ${ }^{8}$

O limite de quantificação (LQ) é definido como a menor concentração do analito de interesse em uma amostra, que pode ser quantitativamente determinado com valores aceitáveis de precisão e exatidão. Alguns autores utilizam o termo limite de quantificação, ${ }^{2,9,15}$ enquanto outros subdividem o termo em limite inferior de quantificação (LIQ) e limite superior de quantificação (LSQ). ${ }^{1,2,6,7,13,49}$

\section{Limite inferior de quantificação (LIQ)}

O LIQ é estabelecido através da análise de amostras branco adicionadas do analito, em concentrações decrescentes, até se obter níveis que sejam quantificados com valores aceitos de precisão e exatidão. Estes valores devem ser maiores que $15 \%$ e menores ou iguais a 20\%. . $^{1,2}$ Além disso, as diretrizes do FDA $^{1}$ e da ANVISA $^{2}$ aceitam que o limite inferior de quantificação seja determinado com relação à menor concentração do analito que produza uma resposta de, no mínimo, 5 vezes a resposta da linha de base obtida pela amostra branco. No entanto, deve-se especificar qual dos dois métodos foi utilizado para a determinação do LIQ. O LIQ pode coincidir com a menor concentração da curva de calibração, sem que seja confundido com o limite de detecção ou controle de qualidade de menor concentração (CQB). ${ }^{49}$

\section{Limite superior de quantificação (LSQ)}

O LSQ é a concentração máxima de analito que pode ser quantificada com valores de precisão e exatidão aceitáveis. De forma geral, o LSQ apresenta o mesmo valor da mais alta concentração utilizada na curva de calibração. ${ }^{15}$

\section{Recuperação (eficiência de extração)}

A recuperação avalia a eficiência do método de tratamento das amostras biológicas. Este parâmetro é calculado comparando-se a resposta obtida para o analito adicionado na matriz biológica e extraído com a resposta obtida para o analito em amostras preparadas em solvente e, conseqüentemente, não extraídas, as quais representam 100\%., 1,2,15

Para a avaliação da recuperação, devem-se utilizar concentrações em três níveis: baixo, médio e alto, de acordo com a curva de calibração. Comumente, utilizam-se as mesmas concentrações empregadas como controles de qualidade do método. . $^{1,2,15}$

Nos métodos bioanalíticos, a recuperação deve ser também avaliada para os compostos utilizados como padrão interno, independente da concentração usada. Aceita-se uma variação de até $15 \%$ do valor de recuperação determinado para os analitos de interesse. ${ }^{13}$

Embora altos valores de recuperação sejam desejáveis para maximizar a sensibilidade do método, não é necessário que este valor seja de $100 \%$ e, sim, que a recuperação seja consistente, precisa e reprodutiva. . $^{1,249}$

Para cálculos de recuperação de amostras derivadas durante o procedimento de extração, faz-se necessária a obtenção de amostra padrão derivada. $^{7}$

Na utilização de métodos bioanalíticos multidimensionais, a recuperação é avaliada em termos da eficiência da transferência da banda cromatográfica de interesse. Este parâmetro é calculado comparando-se as áreas das bandas cromatográficas obtidas da injeção das amostras do analito preparadas em solvente com as das amostras branco adicionada com o analito de interesse. . $7,28,33,34,47,48^{-1}$

\section{Estabilidade}

Para assegurar que a estabilidade do analito seja mantida nas condições de preparo, análise e estocagem das amostras, vários tipos de estabilidade devem ser avaliados durante a validação de um método analítico: ${ }^{1,2,4}$ estabilidade das soluções-padrão, estabilidade após ciclos de congelamento e descongelamento, estabilidade de curta duração, estabilidade das amostras processadas e estabilidade de longa duração.

Recomenda-se avaliar a estabilidade das soluções-padrão em uma única concentração e, no mínimo, em duplicata. ${ }^{4}$ Para os demais estudos, as análises devem ser realizadas em triplicata, com amostras CQ nas concentrações baixa (CQB) e alta (CQA). ${ }^{1,2,7}$ Porém, quando somente três replicatas são analisadas, a probabilidade de conclusões errôneas sobre os dados é alta e, por esse motivo, Hartmann et al. ${ }^{8}$ propõem um mínimo de seis réplicas. 
Para avaliar a estabilidade do analito em uma dada matriz, normalmente três procedimentos experimentais são empregados: ${ }^{14}$ (i) amostras CQ são preparadas e armazenadas nas condições desejadas durante um período de tempo. Depois do período de estocagem, um segundo grupo de amostras CQ, nos mesmos níveis de concentração, é preparado usando solução-estoque recém-preparada. As amostras CQ recém-preparadas são analisadas junto com as amostras armazenadas, usando uma curva de calibração obtida com padrões recémpreparados. Os resultados obtidos com as amostras CQ armazenadas são comparados com aqueles obtidos utilizando-se amostras CQ recém-preparadas; (ii) amostras CQ são preparadas, armazenadas e, depois, analisadas em uma curva de calibração construída com padrões recém-preparados. O analito é considerado estável se um número pré-determinado de amostras CQ cumpre os critérios de aceitação especificados para o método; (iii) um lote de amostras CQ é preparado e algumas amostras são analisadas imediatamente após o preparo em uma curva de calibração gerada com padrões recém-preparados. Outros CQ do mesmo lote são armazenados e quantificados, depois de serem submetidos às condições do estudo de estabilidade, em outra curva de calibração. As concentrações das amostras CQ determinadas em cada ocasião são comparadas para avaliar a estabilidade.

Em relação aos critérios de aceitação dos estudos de estabilidade, algumas considerações são encontradas na literatura. Não há uma concordância sobre o critério de aceitação que define a estabilidade aceitável para as soluções-padrão. Entretanto, o consenso é que a degradação deve ser pequena, uma vez que estas soluções são usadas no preparo dos padrões de calibração e amostras CQ. ${ }^{23}$ Para os demais estudos de estabilidade, as amostras serão consideradas estáveis quando não se observar desvio superior a $10^{8,13}$ ou $15 \% \%^{2,14}$ do valor obtido para as amostras recém-preparadas.

\section{Estabilidade das soluções-padrão}

A estabilidade do analito e do padrão interno nas soluções-padrão estoque, preparadas em concentrações conhecidas e com solventes apropriados, deve ser investigada no início da validação do método, para que seja estabelecido o tempo de validade de tais soluções. Os experimentos devem contemplar a temperatura adequada para o armazenamento (ambiente, sob refrigeração ou congelamento) e o período de estocagem das soluções. As análises devem ser realizadas em intervalos de tempo apropriados, por exemplo, no mínimo $6 \mathrm{~h}$ após o preparo ${ }^{1,2,4}$ e depois de 15 e 30 dias. $^{13}$

Os resultados obtidos com as soluções estoque recém-preparadas devem ser comparados com aqueles obtidos utilizando-se as soluçõespadrão estoque armazenadas. Caso seja confirmada a estabilidade das soluções estoque, os padrões de calibração e as amostras CQ utilizados durante a validação e na aplicação do método analítico podem ser preparados a partir da mesma solução estoque, ${ }^{51}$ sem a necessidade de preparar novas soluções.

\section{Estabilidade após ciclos de congelamento e descongelamento}

Freqüentemente, as amostras do estudo são congeladas e descongeladas para re-análises. Por esse motivo, deve ser avaliada a estabilidade do analito na matriz biológica após, no mínimo, três ciclos de congelamento e degelo. Um experimento para avaliar este tipo de estabilidade é proposto nos guias para validação de métodos bioanalíticos elaborados pelo FDA $^{1}$ e pela $\mathrm{ANVISA}^{2}$ e, também, no artigo de Bansal e DeStefano: ${ }^{4}$ amostras CQ devem ser congeladas na temperatura pretendida para o armazenamento das amostras do estudo (normalmente $\mathrm{a}-20{ }^{\circ} \mathrm{C} \mathrm{ou}-70{ }^{\circ} \mathrm{C}$ ) e mantidas nessa temperatura por $24 \mathrm{~h}$. Depois desse período, as amostras devem ser submetidas ao descongelamento à temperatura ambiente. Quando completamente descongeladas, as amostras devem ser novamente congeladas, na mesma temperatura, por 12 a 24 h e, então, descongeladas. Este ciclo de congelamento e degelo é repetido, no mínimo, mais duas vezes. O analito deve ser quantificado (i) após o último ciclo de congelamento e degelo ${ }^{1,2,4,10,14}$ ou (ii) após cada ciclo. ${ }^{10,14}$ Em ambos os casos, os resultados devem ser comparados com aqueles obtidos nas análises das amostras recém-preparadas ${ }^{1,2,14}$ ou com os valores nominais dos CQ. ${ }^{4}$

Se o procedimento (i) é usado e for constatada a instabilidade do analito, então as amostras CQ devem ser analisadas após cada ciclo de congelamento e degelo, para que seja determinado o número de ciclos para o qual o analito se torna instável. ${ }^{4,10,14}$ Caso seja constatato, por exemplo, que o analito permanece estável somente após um ciclo, as amostras não podem ser congeladas e descongeladas mais de uma vez. Nesse caso, durante a coleta das amostras desconhecidas deve-se armazenar, na temperatura adequada, um número suficientemente grande de alíquotas que permita, se necessário, reanálises.

\section{Estabilidade de curta duração}

Deve ser demonstrado que o analito permanece estável na matriz biológica nas condições do laboratório (temperatura, umidade e luminosidade) e durante o processo de preparo das amostras. É importante ressaltar que todas as etapas envolvidas no preparo da amostra devem ser cuidadosamente avaliadas para verificar se em algum momento o analito sofre degradação. $\mathrm{O}$ exato momento que ocorre a instabilidade deve ser detectado para que sejam tomadas medidas para estabilizar o analito nas etapas que apresentam problemas.

Para avaliar a estabilidade do analito em condições de curta duração, o seguinte procedimento experimental é recomendado: amostras CQ devem permanecer à temperatura ambiente durante várias horas, por exemplo, de 4 a $24 \mathrm{~h}$, baseado no tempo em que as amostras do estudo serão mantidas nessa temperatura. ${ }^{1,2,4,14}$

Os resultados dessa estabilidade devem ser comparados com aqueles obtidos para as amostras CQ recém-preparadas, que são analisadas sem o tempo de espera na bancada, ${ }^{2,14}$ ou com os valores nominais dos respectivos CQ. ${ }^{4}$

Se for constatada a instabilidade do analito nessas condições, temperaturas menores devem ser avaliadas; as amostras, por exemplo, podem ser colocadas em banho de gelo. ${ }^{14}$ Para certos analitos, como captopril e ácido acetilsalicílico, por exemplo, que sofrem alteração/ degradação na matriz biológica, aditivos apropriados (inibidores enzimáticos, anti-oxidantes, etc.) devem ser adicionados nas amostras com o intuito de estabilizá-los no fluido biológico. ${ }^{10}$

Caso a diminuição da temperatura e o uso de aditivos não sejam suficientes para reduzir a degradação do analito na matriz biológica, o seguinte procedimento deve ser avaliado: ${ }^{10,14}$ imediata extração do analito da matriz biológica e congelamento do resíduo seco, para posterior reconstituição e análise.

\section{Estabilidade das amostras processadas}

Problemas de instabilidade podem ocorrer não somente na matriz biológica e/ou durante o tratamento das amostras, mas também nas amostras processadas. Portanto, é necessário testar a estabilidade do analito no solvente em que foram extraídos ou reconstituídos, na temperatura do auto-injetor e também em temperaturas mais baixas (sob refrigeração), caso os extratos das amostras sejam armazenados antes das análises. Em relação ao tempo, o mesmo deve ser superior ao esperado para a duração da análise cromatográfica, o que possibilita, se necessário, a reinjeção dos extratos ou um tempo de espera devido a circunstâncias não previstas como, por exemplo, o mau funcionamento do sistema cromatográfico. ${ }^{7,10,14}$

Dadgar et al. ${ }^{10} \mathrm{e}$ Wieling et al. ${ }^{50}$ propõem que as replicatas das extrações de cada concentração CQ sejam misturadas, agitadas e 
aliquotadas para injeções nos tempos zero, intermediário (a cada $2 \mathrm{~h}$ ) e máximo programado para completar o estudo (por exemplo, 24, 36 ou 48 h). A comparação das concentrações das amostras no tempo zero e demais tempos será independente da reprodutibilidade da extração, quando se trabalha com a mistura das frações extraídas em cada concentração.

A avaliação da estabilidade das amostras após processamento com padrões de calibração recém-preparados não é um procedimento normalmente utilizado na validação, mas pode ser conduzido. ${ }^{23} \mathrm{Um}$ exemplo de como este tipo de estabilidade pode ser avaliada é apresentada por Wieling et al.. ${ }^{50}$

\section{Estabilidade de longa duração}

Dois problemas podem ocorrer quando amostras biológicas são armazenadas por longos períodos de tempo: a adsorção do analito no recipiente e/ou a degradação dos compostos de interesse, com subseqüente formação de metabólitos e/ou outras substâncias. Por esse motivo, a estabilidade de longa duração do analito na matriz biológica deve ser estabelecida nas mesmas condições de estocagem das amostras desconhecidas para o qual o método analítico está sendo desenvolvido, ${ }^{1,2,4,10,14}$ ou seja, (i) no mesmo tipo de recipiente e na mesma temperatura em que as amostras do estudo serão armazenadas; (ii) por um período de tempo superior ao intervalo de tempo compreendido entre a coleta da primeira amostra do estudo e a análise da última. As amostras devem ser analisadas em vários tempos, por exemplo, 1, 3, 6, 9 e 12 meses, dependendo da estabilidade requerida.

Idealmente, as amostras controle usadas para avaliar a estabilidade de longa duração devem ser coletadas dos pacientes/ voluntários, misturadas, aliquotadas e armazenadas nas mesmas condições a que as amostras do estudo serão submetidas. Entretanto, o uso de amostras CQ preparadas com uma matriz biológica isenta do analito (matriz branco) é uma alternativa aceitável e mais comumente utilizada. ${ }^{10}$

Dadgar et al. ${ }^{14}$ propõem que a estabilidade de longa duração do analito na matriz biológica seja avaliada concomitantemente com a estabilidade de uma matriz branco, para determinar se a degradação da matriz acarreta em um aumento de interferências. Este procedimento foi adotado por Oliveira e $\mathrm{Cass}^{47}$ no desenvolvimento e validação de um método analítico, usando injeção direta de amostra, para quantificar cefoperazone em leite bovino. As análises realizadas para avaliar a estabilidade da matriz biológica mostraram que a mesma permaneceu estável por um período de 3 dias, quando armazenada a 4 ou $-20{ }^{\circ} \mathrm{C}$

Para verificação da estabilidade de longa duração, réplicas de ambas as amostras CQ, recém-preparadas e estocadas, são analisadas e suas respostas comparadas. ${ }^{52-54}$

\section{Validação da diluição}

Se for esperado que a concentração de algumas amostras desconhecidas possa exceder a do maior padrão da curva de calibração, a integridade da diluição dessas amostras deve ser demonstrada. ${ }^{1,2,4,10}$ Para isso, amostras CQ preparadas em concentração várias vezes maior que o limite superior da curva de calibração devem ser diluídas com uma matriz branco e analisadas, no mínimo, em um dia da validação. Os critérios de aceitação para as amostras CQ diluídas são os mesmos estabelecidos para os parâmetros de exatidão e precisão do método. ${ }^{1,4,10}$

É importante salientar que se durante as análises das amostras do estudo for constatada a necessidade de uma diluição maior que aquela anteriormente avaliada, uma outra validação da diluição deve ser realizada. ${ }^{4}$

\section{Robustez}

A robustez de um método analítico mede sua suscetibilidade frente a pequenas variações que podem ocorrer durante as análises de rotina. Os testes de robustez são de fundamental importância para que os analistas conheçam quais fatores devem ser estritamente controlados durante a execução de um método. Para examinar as causas de variabilidade dos resultados, vários fatores devem ser avaliados, tais como: $\mathrm{pH}$, força iônica e quantidade do modificador orgânico da fase móvel, temperatura da coluna, vazão da fase móvel, diferentes colunas (diferentes lotes e/ou fabricantes), procedimentos envolvidos no preparo das amostras, etc. Se as alterações das condições de análise produzirem resultados dentro dos limites aceitáveis de seletividade, exatidão e precisão, o método pode ser considerado robusto e tais variações podem ser incorporadas ao procedimento. ${ }^{31}$ Por outro lado, constatando-se susceptibilidade do método a tais variações, estas deverão ser adequadamente controladas, ou precauções deverão ser incluídas no procedimento. ${ }^{2}$

Vander-Heyden et al..$^{55}$ apresentam uma excelente discussão sobre todas as etapas envolvidas em um estudo de robustez: identificação e seleção dos fatores a serem investigados, definição dos diferentes níveis para os fatores, seleção e execução dos experimentos, determinação das respostas, análises estatística e/ou gráfica dos efeitos, etc. Nesse artigo, também é descrito um estudo de caso para exemplificar o estudo da robustez.

\section{CONCLUSÃO}

A validação de métodos bioanalíticos aplicados a estudos farmacocinéticos, de biodisponibilidade, bioequivalência ou para a quantificação de resíduos, baseia-se na interpretação das diretrizes de diferentes agências reguladoras, apresentadas e discutidas nesta revisão. Os trabalhos reportados, assim como as diretrizes apresentadas, mostram que existe um consenso sobre as principais figuras de mérito e os critérios de aceitação que devem ser considerados para a creditação dos resultados obtidos em um método bioanalítico, embora não haja consenso em alguns dos procedimentos de avaliação de algumas das figuras de mérito. Considerando o progressivo desenvolvimento tecnológico, associado aos métodos bioanalíticos, as normas para validação têm sido constantemente revistas e novos parâmetros incluídos, de maneira a contribuir para a evolução e expansão das ferramentas bioanalíticas. Essa revisão contempla os atuais conceitos de validação.

\section{GLOSSÁRIO}

As definições dos termos utilizados neste artigo foram extraídas do glossário relativo aos métodos analíticos e bioanalíticos da ANVISA. ${ }^{2}$

Amostra branco: amostra de uma matriz biológica isenta do analito em estudo.

Amostra controle de qualidade (CQ): amostra da matriz biológica adicionada do analito que é utilizada para monitorar o desempenho de um método bioanalítico e para avaliar a integridade e validade dos resultados das amostras desconhecidas analisadas.

Amostra processada: extrato final (anterior à análise instrumental) de uma amostra que foi submetida a várias manipulações (extração, diluição, concentração, etc).

Amostra desconhecida: amostra biológica que é objeto de análise.

Corrida analítica: conjunto completo de amostras em estudo com um número apropriado de padrões e CQs para sua validação. Várias corridas podem ser realizadas em um dia ou uma corrida analítica pode demorar vários dias para ter sua análise concluída. 
Padrão de calibração: matriz biológica na qual foi adicionada uma quantidade conhecida do analito. Os padrões de calibração são usados para construir a curva de calibração a partir da qual são determinadas as concentrações do analito nas amostras CQ e nas amostras desconhecidas em estudo.

Padrão interno (PI): composto quimicamente estável, geralmente com estrutura química similar à do analito, que é adicionado aos padrões de calibração e amostras em concentrações conhecidas e constantes para facilitar a quantificação do analito.

\section{REFÊRÊNCIAS}

1. United States Food and Drug Administration (US-FDA); Guidance for Industry, Bioanalytical Method Validation, 2001.

2. Agência Nacional de Vigilância Sanitária (ANVISA); Guia para Validação de Métodos Analíticos e Bioanalíticos, Resolução RE no 899 de 29/05/2003.

3. Official Journal of the European Communities (OJEC); Implementing Council Directive 96/23/EC concerning the performance of analytical methods and the interpretation of results, 08/2002.

4. Bansal, S.; DeStefano, A.; AAPS J. 2007, 9, E109.

5. Shah, V. P.; AAPS J. 2007, 9, E43.

6. Peters, F. T.; Drummer, O. H.; Musshoff, F.; For. Sci. Int. 2007, 165, 216.

7. Peters, F. T.; Maurer, H. H.; Accred. Qual. Assur. 2002, 7, 441.

8. Hartmann, C.; Smeyers-Verbeke, J.; Massart, D. L.; McDowall, R. D.; J. Pharm. Biomed. Anal. 1998, 17, 193.

9. Bressolle, F.; Bromet-Petit, M.; Audran, M.; J. Chromatogr., B: Anal. Technol. Biomed. Life Sci. 1996, 686, 3.

10. Dadgar, D.; Burnett, P. E.; Choc, M. G.; Gallicano, K.; Hooper, J. W.; J. Pharm. Biomed. Anal. 1995, 13, 89.

11. Lang, J. R.; Bolton, S.; J. Pharm. Biomed. Anal. 1991, 5, 357.

12. Buick, A. R.; Doig, M. V.; Jeal, S. C.; Land, G. S.; McDowall; R. D.; J. Pharm. Biomed. Anal. 1990, 8, 629.

13. Causon, R.; J. Chromatogr., B: Anal. Technol. Biomed. Life Sci. 1997, 689, 175.

14. Dadgar, D.; Burnett, P. E.; J. Pharm. Biomed. Anal. 1995, 14, 23.

15. Shah, V. P.; Midha, K. K.; Dighe, S.; McGilveray, I. J.; Skelly, J. P.; Yacobi, A.; Layloff, T.; Viswanatham, C. T.; Cook, C. E.; McDowall, R. D.; Pittman, K. A.; Spector, S.; Pharm. Res. 1992, 9, 588.

16. Hill, H. M.; Chromatographia 2000, 52, 65.

17. Matuszewski, B. K.; Constanzer, M. L.; Chavez-Eng, C. M.; Anal. Chem. 2003, 75, 3019.

18. Tang, L.; Kebarle, P.; Anal. Chem. 1991, 63, 2709.

19. Taylor, P. J.; Clin. Biochem. 2005, 38, 328.

20. King, R.; Bonfiglio, R.; Fernandez-Metzler, C.; Miller-Stein, C.; Olah, T.; J. Am. Soc. Mass Spectrom. 2000, 11, 942.

21. Crotti, A. E. M.; Vessechi, R.; Lopes, J. L. C.; Lopes, N. P.; Quim. Nova 2006, 2, 287.

22. Bonfiglio, R.; King, R. C.; Olah, T. V.; Merkle, K.; Rapid Commun. Mass Spectrom. 1999, 13, 1175.

23. Viswanathan, C. T.; Bansal; S.; Booth, B.; DeStefano, A. J.; Rose, M. J.; Sailstad, J.; Shah, V. P.; Skelly, J. P.; Swann, P. G.; Weiner, R.; AAPS J. 2007, 9, E30.

24. Souverain, S.; Rudaz, S.; Veuthey, J. L.; J. Chromatogr, A 2004, 1058, 61.

25. Georgi, K.; Boos, K. S.; Chromatographia 2006, 63, 523.
26. Wang, Z.; Wen, J.; He, Y.; J. Pharm. Biomed. Anal. 2007, 45, 145.

27. Cass, Q. B.; Degani, A. L. G.; Cassiano, N. M.; Pedrazolli Jr., J.; J. Chromatogr., B: Anal. Technol. Biomed. Life Sci. 2002, 766, 153.

28. Cass, Q. B.; Lima, V. V.; Oliveira, R. V.; Cassiano, N. M.; Degani, A. L.; Pedrazzoli Jr., J.; J. Chromatogr., B: Anal. Technol. Biomed. Life Sci. 2003, 798, 275.

29. Xu, R. N.; Fan. L.; Rieser, M. J.; El-Shourbagy, T. A.; J. Pharm. Biomed. Anal. 2007, 44, 342.

30. Rogatsky, E.; Stein, D.; J. Am. Soc. Mass Spectrom. 2005, 16, 1757.

31. Ribani, M.; Bottoli, C. B. G.; Collins, C. H.; Jardim, I. C. S. F.; Melo, L. F. C.; Quim. Nova 2004, 27, 771.

32. Cuadros-Rodríguez, L.; Gámiz-Gracia, L.; Almansa-López, E. M.; Bosque-Sendra, J. M.; Trends Anal. Chem. 2001, 20, 620.

33. Cass, Q. B.; Galatti, T. F.; J.Pharm. Biomed. Anal. 2008, 46, 937.

34. Cass, Q. B.; Gomes, R. F.; Calafatti, S. A.; Pedrazolli Jr, J.; J. Chromatogr., A 2003, 987, 235.

35. Ellison, S. L. R.; Thompson, M.; Analyst 2008, 133, 992.

36. Almeida, A. M.; Castel-Branco, M. M.; Falcão, A. C.; J. Chromatogr., B; Anal. Technol. Biomed. Life Sci. 2002, 774, 215.

37. Singtoroj, T.; Tarning, J.; Annerberg, A.; Ashton, M.; Bergqvist, Y.; White, N. J.; Lindegardh, N.; Day, N. P. J.; J. Pharm. Biomed. Anal. 2006, 41, 219.

38. Barros Neto, B.; Scarminio, I. S.; Bruns, R. E.; Planejamento e Otimização de Experimentos, $3^{\mathrm{a}}$ ed., Ed. da UNICAMP: Campinas-SP, 2007.

39. Chui, Q. S.; Zucchini, R. R.; Lichtig, J.; Quim. Nova 2001, 24, 374.

40. Ribeiro, F. A. L.; Ferreira, M. M. C.; Morano, S. C.; Silva, L. R.; Schneider, R. P.; Quim. Nova 2008, 31, 164.

41. Custódio, R.; Andrade, J. C.; Augusto, F.; Quim. Nova 1997, $20,219$.

42. Pimentel, M. F.; Barros Neto, B.; Quim. Nova 1996, 19, 268.

43. Kohler, M.; Haerdi, W.; Chrosten, P.; Veuthey, J. L.; Trends Anal. Chem. 1997, 16, 475.

44. Shabir, G. A.; J. Chromatogr., A 2003, 987, 57

45. International Conference on Harmonization (ICH); Guidance for Industry Q2A: Text on Validation of Analytical Procedures, 1995.

46. Beltrame, F. L.; Rodrigues Filho, E.; Barros, F. A. P.; Cortez, D. A. G.; Cass, Q. B.; J. Chromatogr., A 2006, 1119, 257.

47. Oliveira, R. V.; Cass, Q. B., J. Agric. Food Chem. 2006, 54, 1180.

48. Oliveira, R. V.; De Pietro, A. C.; Cass, Q. B.; Talanta 2007, 71, 1233.

49. Shah, V. P.; Midha, K. K.; Findlay, J. W.; Hill, H. M.; Hulse, J. D.; McGilveray, I. J.; McKay, G.; Miller, K. J.; Patnaik, R. N.; Powell, M. L.; Tonelli, A.; Viswanatham, C. T.; Yacobi, A.; Pharm. Res. 2000, 17, 1551.

50. Wieling, J.; Hendriks, G.; Tamminga, W. J.; Hempenius, J.; Mensink, C. K.; Oosterhuis, B.; Jonkman, J. H.; J. Chromatogr., A 1996, 730, 381.

51. Brodie, R. R.; Hill, H. M.; Chromatographia 2002, 55, S-91.

52. Pereira, A. V.; Cass, Q. B.; J. Chromatogr., B: Anal. Technol. Biomed. Life Sci. 2005, 826, 139.

53. Massaroti, P.; Cassiano, N. M.; Duarte, L. F.; Campos, D. R.; Marchioretto, M. A. M.; Bernasconi, G.; Calafatti, S. A.; Barros, F. A. P.; Meurer, E. C.; Pedrazzoli Jr., J.; J. Pharm. Pharmaceut. Sci. 2005, 8, 340.

54. Massaroti, P.; Moraes, L. A. B.; Marchioretto, M. A. M.; Cassiano, N. M.; Bernasconi, G.; Calafatti, S. A.; Barros, F. A. P.; Meurer, E. C.; Pedrazzoli Jr., J.; Anal. Bioanal Chem. 2005, 382, 1049.

55. Vander-Heyden, Y.; Nijhuis, A.; Smeyers-Verbeke, J.;Vandeginste, B. G. M.; Massart, D. L.; J. Pharm. Biomed. Anal. 2001, 24, 723. 